\title{
Evaluation of Urinalysis Parameters to Predict Urinary-Tract Infection
}

\author{
Juliana Conrad dos Santos ${ }^{1}$, Liliana Portal Weber ${ }^{1}$ and Leandro Reus Rodrigues Perez ${ }^{2,3}$ \\ ${ }^{1}$ Faculty of Pharmacy, Caxias do Sul University; ${ }^{2}$ Microbiology Unit, Mãe de Deus Hospital; ${ }^{3}$ Pharmaceutical Sciences Postgraduate Program, \\ Federal University of Rio Grande do Sul (UFRGS); Porto Alegre, RS, Brazil
}

\begin{abstract}
We evaluated the performance of automated-flow cytometry, urinalysis dipsticks and microscopic urine sediment analysis as predictors of urinary tract infection. Urine cultures were used as a reference method for comparison. Six-hundred-seventy-five urine samples from hospitalized and not hospitalized patients attended at Hospital Mãe de Deus, Porto Alegre, in south Brazil, were included in the study. Among the individual measures analyzed, intense bacteriuria in the microscopic analysis of urinary sediment gave an accuracy of $92.9 \%$. A combination between intense bacteriuria (microscopic analysis) and $>20$ leukocytes per $\mu \mathrm{L}$ of urine (flow cytometry) gave a higher accuracy (97.3\%). We conclude that though it is laborious, microscopic urinalysis is a good analytical tool. Taken together with flow cytometry and dipsticks, we obtained a clinically-acceptable prediction of urinary-tract infection.
\end{abstract}

Key-Words: Urinary tract infection, urinalysis, flow cytometry.

Urinary tract infection (UTI) is a term applicable to a great variety of clinical conditions, from asymptomatic bacteriuria to severe infections, as for example, sepsis. UTI is a medical problem that is quite frequent in the population [1]. In most cases, rapid tests are used for initial treatment. Urinalysis is one of the most important tests used in clinical laboratories in the diagnosis and follow-up of UTI [2]. Microscopic-urine-sediment analysis has been the methodology most used for examining urine cells, particles and microorganisms. However, it is labor-intensive and time consuming; there is also considerable interobserver variability. Chemical screening with dipstick reagents has been widely used as complement to urinalysis exams. A flow-cytometrybased walkaway instrument, the Sysmex UF-100 (Sysmex Corporation, Japan), which performs automated microscopic urinalysis, facilitates this type of analysis [3,4]. Urinalysis automation improves both the accuracy and the productivity of urine-sediment analysis for urine screen evaluation, especially to predict the growth of microorganisms in culture. However, microscopic urine inspection is necessary for cases in which glomerular tube diseases are suspected. In these cases, visualization of pathologic cylinders and erythrocyte dysmorphism is essential for diagnosis.

The feasibility of a flow-cytometry urinalysis for selecting samples that require microscopic examination has been previously discussed [2-5]. We evaluated the individual parameters and crosschecked Sysmex UF-100 data with results obtained with dipstick testing and microscopic-inspection urinalysis to predict UTI, using the results of urine culture as a reference method. We also examined the prevalence of microorganisms causing UTI.

Received on 12 February 2007; revised 7 August 2007.

Address for correspondence: Dr. Leandro Reus Rodrigues Perez. Laboratory of Clinical Analysis, Faculty of Pharmacy, UFRGS, Ipiranga Avenue 2752/303, Porto Alegre, RS, Brazil. Zip code: 90610-000. Phone: +55 (51) 33085412. Fax: +55 (51) 33085437. E-mail: leandro.reus@gmail.com.

The Brazilian Journal of Infectious Diseases 2007;11(5):479-481. (C) 2007 by The Brazilian Journal of Infectious Diseases and Contexto Publishing. All rights reserved.

\section{Materials and Methods}

Urine Specimens

We evaluated 675 urine specimens submitted to our laboratory for diagnostic urinalysis during August 2006. Only samples for which cultures were solicited were included in our study. Most of the urine samples were obtained by the midstream technique (recommended). All samples were submitted to flow cytometry, chemical dipstick tests and culture. Only samples that were found to have parameters different from normal were submitted to microscopic sediment analysis.

\section{Flow Cytometry by Sysmex UF-100}

The Sysmex UF-100 is an automated analyzer that uses argon-laser flow cytometry. The UF-100 measures conductivity and categorizes the particles on the basis of their shape, size, volume, and staining characteristics. Briefly, after identifying the specimen by its bar code, the UF-100 aspirates $800 \mu \mathrm{L}$ of urine and performs the analysis. The results are displayed in scattergrams, histograms, and counts per microliter, as well as counts per high-power field (HPF). The upper reference limits for microscopy used in our laboratory are as follows: leukocytes, $<20$ per $\mu \mathrm{L}$ of urine and epithelial cells, $<30$ per $\mu \mathrm{L}$ of urine. Problematic specimens are submitted to microscopic analysis.

\section{Dipstick Urinalysis}

Dipstick urinalysis was done using Combur 10-Test M strips and a Miditron automated-reflectance photometer (Roche Diagnostics, São Paulo, Brazil). The strips had reagent pads for semiquantitative assessment of density, $\mathrm{pH}$, leukocyte esterase, nitrite, protein, glucose, ketones, urobilogen, bilirubin, and hemoglobin/mioglobin. As a predictive parameter for UTI, we evaluated leukocyte esterase $(3+)$ and nitrite reaction.

\section{Microscopic Sediment Urinalysis}

Manual microscopic sediment inspection was performed as follows: each urine sample $(10 \mathrm{~mL})$ was centrifuged at $1,500 \mathrm{x}$ 
for $5 \mathrm{~min}$, and the supernatant was removed. At least 20 random microscopic fields were examined at X400 (HPF) for each sample, and the mean number of cells and particles/HPF were calculated. To convert from elements/HPF to elements/ $\mu \mathrm{L}$, we adopted a multiplication factor of five. Bacteriuria was classified as moderate or intense for each sample.

\section{Culture Conditions and Medium}

All samples submitted to urinalysis were also cultured. The culture was done quantitatively, being strap calibrated $(1 \mu \mathrm{L})$. Blood agar and MacConkey agar plates were purchased from Newprov (Pinhais, PR, Brazil). The cultures were read after 24 hours of incubation at $35^{\circ} \mathrm{C}$. The minimum microbial growth considered was $10^{3}$ colony-forming units (CFU) per $\mathrm{mL}$ of urine. Samples that grew more than one microorganism, with counts inferior to $10^{5} \mathrm{CFU}$ per $\mathrm{mL}$ of urine, were classified as mixed growth and were considered inadequate for analytical purposes.

\section{Bacterial Identification}

For Gram-negative rods, identification was done using the GNI card of the automated system Vitek 1 (Biomerieux, Marcy l'Etoile, France). For Staphylococcus saprophyticus identification, we used a coagulase tube test and resistance to a $10 \mu \mathrm{g}$ novobiocin disk (Oxoid, Hampshire, UK). For Streptococcus agalactiae identification, we used a campfactor test. For Staphylococcus aureus, a coagulase tube test was utilized. Enterococcus spp. were identified to the genus level, using a bile-esculin test and pyrrolidonylarylamidase (PYR) from Laborsys (Porto Alegre, RS, Brazil). The Streptococcus spp. viridans group was considered when bileesculin and PYR tests were negative.

\section{Statistical Analysis}

The statistical significance of the data was determined by calculating the $P$-values with the exact Fisher's test. P-values $<0.0001$ were considered statistically significant.

\section{Results}

Among the 675 urine samples analyzed, 79.6\% (537/675) were female patients and $20.4 \%$ (138/675) were male patients. Among the 675 cultures, 81.5\% (550/675) did not give bacterial growth, 16\% (108/675) showed bacterial growth, and 2.5\% $(17 / 675)$ were classified as inadequate samples. Among the 108 urine samples that gave growth in culture, 83\% (90/108) yielded more than $10^{5} \mathrm{CFU}$ per $\mathrm{mL}$ of urine.

Among 108 cultures with microbial growth, 86\% (93/108) were from female patients and $14 \%(15 / 108)$ were from male patients. The prevalence of microorganisms in female patients was: Escherichia coli (67\%; 62/93), S. saprophyticus (11\%; 10/93), S. agalactiae, Enterococcus spp. and Klebsiella pneumoniae (4.3\%; 4/93), Proteus mirabilis (3.2\%; 3/93), S. aureus and Streptococus spp. viridans group (2.1\%; 2/93), and Enterobacter cloacae (1.1\%; 1/93). In male patients, the prevalence was: E. coli ( 10/15), and Enterococcus spp.,
Citrobacter koseri, P. maribilis, Enterobacter cloacae and S. agalactiae $(1 / 15)$.

The sensitivity, specificity, predictive values (positive and negative) and accuracy for the parameters analyzed as predictors of UTI are shown in Table 1.

\section{Discussion}

Urinalysis is a high-volume procedure that normally requires significant labor. However, urinalysis parameters are still extensively used to guide empirical treatment of urinarytract infections.

In our study, $79.6 \%$ of the patients were female. McLaughlin \& Carson (2004) indicated a higher prevalence of UTI in female patients due to anatomic and physiological factors (proximity of the urethra with the vagina and the rectum). In female patients, the most prevalent microorganism was E. coli (67\%), followed by S. saprophyticus (9.3\%). Although less prevalent than E. coli, S. saprophyticus infections are more aggressive, often affecting the upper urinary tract, with a high probability of recurrent infections, normally affecting about half of the female patients with UTI $[7,8]$.

Among the cultures that gave growth, 83\% gave counts superior to $10^{5} \mathrm{CFU}$ per $\mathrm{mL}$ of urine. Normally, growth superior to $10^{5} \mathrm{CFU}$ per $\mathrm{mL}$ of urine indicates UTI. However, UTI is more conclusive if the patient has clinical symptoms and leukocytes in the urine [9]. In our study, 68\% (61/90) of the samples presented counts above 20 leukocytes per $\mu \mathrm{L}$ of urine. Among the parameters analyzed as predictors of UTI, intense bacteriuria gave the highest predictive power (92.9\%). However, the laborious, microscopic-urinary-sediment analysis for bacteriuria was also found to be an important parameter to predict UTI.

Association of the parameters intense bacteriuria and counts $>20$ leukocytes per $\mu \mathrm{L}$ of urine gave an accuracy of $97.3 \%$. A limitation of this type of analysis based on urine sediment is interobserver variability.

Other combinations of parameters were also compared, and the combinations were always superior to single analyses. As an example, a combination of leukocyte esterase (3+) with nitrite positivity (both diagnosed with dipstick reagents) gave an accuracy of $95.6 \%$, while leukocyte esterase (3+) and nitrite positivity, separately, gave $89.9 \%$ and $89.5 \%$, respectively.

In our study, nitrite positivity gave a specificity and a sensitivity of 99.5 and 38.9\%, respectively. Leukocyte esterase (3+) gave a sensitivity and a specificity of $65.4 \%$ and $94 \%$, respectively. When these parameters were combined, we obtained a specificity of $100 \%$ and a sensitivity of $55.1 \%$. The higher specificity makes these parameters useful to predict cases of significant growth in culture. These results are similar to those of previous studies $[3,6]$. The nitrite test proved be a specific but relatively insensitive test. The low sensitivity and high specificity presented by the nitrite test make it important in cases in which culture is negative. Some microorganisms that cause UTI, such as enterococci and $S$. 
Table 1. Sensitivity, specificity, positive and negative predictive values and accuracy for parameters analyzed to predict urinary tract infections

\begin{tabular}{|c|c|c|c|c|c|c|}
\hline Parameters & Sensitivity (\%) & Specificity (\%) & VPP (\%) & VPN (\%) & Accuracy (\%) & p value* \\
\hline Nitrite & 38.9 & 99.5 & 93.3 & 89.2 & 89.5 & $<0.0001$ \\
\hline Leukocyte esterase $(3+)$ & 65.4 & 94 & 64.6 & 94.2 & 89.9 & \\
\hline Intense bacteriuria & 52.6 & 98.8 & 87 & 93.4 & 92.9 & \\
\hline$>20$ leukocytes per $\mu \mathrm{L}$ & 83.3 & 76.3 & 38.7 & 96.2 & 77.4 & \\
\hline Nitrite and leukocyte esterase (3+) & 55.1 & 100 & 100 & 95.3 & 95.6 & \\
\hline Nitrite and intense bacteriuria & 43.3 & 100 & 100 & 93.7 & 94 & \\
\hline Nitrite and $>20$ leukocytes per $\mu \mathrm{L}$ & 70.7 & 100 & 100 & 96.7 & 97 & \\
\hline $\begin{array}{l}\text { Leukocyte esterase }(3+) \text { and } \\
\text { intense bacteriuria }\end{array}$ & 62.9 & 99.8 & 95.7 & 97.1 & 97 & \\
\hline $\begin{array}{l}\text { Leukocyte esterase }(3+) \text { and } \\
>20 \text { leukocytes per } \mu \mathrm{L}\end{array}$ & 76.6 & 93.5 & 61 & 96.8 & 91.5 & \\
\hline Intense bacteriuria and & & & & & & \\
\hline >20 leukocytes per $\mu \mathrm{L}$ & 82.4 & 98.7 & 84.9 & 98.4 & 97.3 & \\
\hline
\end{tabular}

*P-value calculated by Fisher's exact test for analysis of a 2x2 contingency table.

saprophyticus, are unable to reduce nitrate to nitrite. Falsenegative results may occur due to frequent urinations, which lower the exposure of the microorganisms to nitrate; this can also occur with a diet poor in vegetables (a source of nitrates) [9].

Only $36.8 \%$ of the samples with counts above 20 leukocytes per $\mu \mathrm{L}$ presented microbial growth in urine culture. An abnormal amount of pus cells in the urine, e.g. polymorphonuclear leukocytes, indicates that an inflammatory response is occurring somewhere in the urinary tract. As with other parameters, pyuria must be interpreted in relation to other clinical and laboratory information. Although UTI are undoubtedly the most common cause of pyuria, other important conditions must be considered. Pyuria is a parameter that is generally associated with infection. Pyuria can occur due to leucorrhea, fever, pregnancy and administration of adrenocortical steroids, in female patients without infection $[7,10]$. For this reason, it is particularly important to understand the natural history and clinical significance of UTI and the relation between pyuria-dysuria syndrome and infection of the urinary tract.

Seventeen urine samples were classified as inadequate. Among these, 13 were obtained from female patients. Mixed growth in female patients can be explained, in part, by vaginal flora contaminants, especially when hygiene is inadequate; although in our study this fact could be masked by the larger number of female patients.

Urinalysis techniques are extensively used in routine laboratories. Microscopic sediment analyses and dipstick chemical tests have long been used for examining urine for infectious processes. However, a flow cytometry urinalysis analyzer performs the operation in a more time and labor-saving manner. Additionally, increased throughput and decreased microscopy review rates are important advantages of this system.

We conclude that urinalysis methods are good predictors of urine-culture diagnosis and can be used as predictors of UTI. Individually, parameters such as intense bacteriuria, leukocyte esterase (3+) and nitrite had good predictive power. An association among the different urinalysis techniques improved accuracy over single analyses.

\section{References}

1. Tanagho E.A., McAninch J.W. Smith's general urology. Sixth edition. Lange Medical Books, NY, 2004.

2. Delanghe J.R., Kouri T.T., Huber A.R., et al. The role of automated urine particle flow cytometry in clinical practice. Clin Chim Acta 2000;301:1-18.

3. Lun A., Ziebig R., Priem F., et al. Routine workflow for use of urine strips and urine flow cytometer UF-100 in the hospital laboratory. Clin Chem 1999;45:1305-7.

4. Ben-Ezra J., Bork L., McPherson R.A. Evaluation of the Sysmex UF-100 automated urinalysis analyzer. Clin Chem 1998;44:92-5.

5. Ottiger C., Huber A.R. Quantitative urine particle analysis: integrative approach for the optimal combination of automation with UF-100 and microscopic review with KOVA cell chamber. Clin Chem 2003;49:617-23.

6. McLaughlin S.P., Carson C.C. Urinary Tract Infections in women. Med Clin N Am 2004;88:417-29.

7. Eiff C., Peters G., Heilmann C. Pathogenesis of infections due to coagulase-negative staphylococci. Lancet 2002;2:677-84.

8. Mehnert-Kay S.A. Diagnosis and Management of Uncomplicated Urinary Tract Infections. American Family Physician 2005;73:451-6.

9. Oplustil C.P., Zoccoli C.M., Tobouti N.R., et al. Procedimentos Básicos em Microbiologia Clínica. Segunda edição. Sarvier, SP, 2004.

10. Guidoni E.B.M., Toporovski J. Infecção Urinária na Adolescência. Jornal de Pediatria 2001;77:165-9. 